\section{PO-0648 NORWEGIAN - INDIAN COLLABORATION PROJECT TO IMPROVE NEWBORN CARE}

${ }^{1} \mathrm{~S}$ Steinnes, ${ }^{2} \mathrm{M}$ Hornslien. ${ }^{1} \mathrm{NICU}$, Oslo University Hospital Ullevaal, Oslo, Norway, ${ }^{2}$ International Collaboration Unit, Oslo University Hospital Ullevaal, Oslo, Norway

\subsection{6/archdischild-2014-307384.1289}

Background Norway India Partnership Initiative (NIPI) is cooperation between the Governments of Norway and India, and has a particular focus on improving newborn health by training health personnel. A former exchange program in Kolkata (20112013) reports reduction in neonatal mortality rate and improved newborn care.

Aim This project aims to improve the care of sick and premature newborns, in line with international standards, in Rajasthan, India. The exchange of health personnel in north-south collaboration can reduce neonatal morbidity and mortality by improving basic neonatal nursing. Focus areas are KMC, nutrition and breastfeeding, developmental care, hygiene and pain management. The project intends to increase multicultural understanding within newborn health care.

Method Exchange of 4 indian and 2 norwegian nurses/year for a 3 year period. Norwegian nurses will stay in JK Lone Hospital, Jaipur for 12 months and indian nurses in Oslo university hospital for 6 months.

Focus for Norwegian nurses in Jaipur will be teaching doctors and nurses in evidence based newborn care through bedside training in the NICU. Interact with the indian team of doctors, nurses, and family of the sick newborn, in order to develop an understanding of the culture and society they are working in.

Focus for Indian nurses in Oslo will be hands on bedside training in the NICU under surveillance of experienced personell. In depth knowledge of the focus areas. All participants are to convey their experience to their home partners after homecoming through teaching, demonstrations and reports. The goal is sustainable practice change according to evidence based care.

\section{P0-0649 USEFULNESS OF POSTMORTEM IMAGING AND AUTOPSY IN NEONATAL INTENSIVE CARE UNIT}

T Sugiura, S Yoshida, N Sato, H Ueda, T Goto, K Ito, R Nagasaki, T Kato, S Saitoh. Pediatrics and Neonatology, Nagoya City University Graduate School of Medical Sciences, Nagoya, Japan

\subsection{6/archdischild-2014-307384.1290}

Background and aims Rates of traditional medical autopsy are low in Japan. In particular, obtaining consent for autopsy of children from parents is difficult. Although postmortem imaging of adults has been well studied, this is not the case in children. Few studies have investigated the accuracy of postmortem imaging in diagnosing the causes of neonatal deaths. We aimed to identify the accuracy of postmortem computed tomography (CT), and compared postmortem CT findings to clinical diagnosis and autopsy in the neonatal intensive care unit (NICU).

Methods Twenty-five patients died in our NICU from 2010 to 2012. Consent for autopsy was obtained for 10 cases (40\%) and consent for postmortem imaging was obtained for 19 (76\%). Both postmortem imaging and autopsy were able to be performed for 10 cases (40\%).

Results The concordance rate between cause of death from postmortem CT and that from clinical diagnosis was 74\% (14/19), while the rate between cause of death from postmortem CT and that from autopsy was 70\% (7/10). Moreover, postmortem CT uncovered unrecognised diagnoses such as pericardial emphysema that remained undetected from the clinical course or autopsy.

Conclusions Postmortem imaging is reliable and valid in NICU settings, and the combination of postmortem imaging and autopsy could improve the accuracy of determining causes of neonatal deaths.

\section{PO-0650 THE INFLUENCE OF DISORDERED EATING ATTITUDES ON PREGNANCY AND NEONATAL OUTCOMES}

${ }^{1}$ B Czech-Szczapa, ${ }^{2} \mathrm{~T}$ Szczapa, ${ }^{3} \mathrm{TA}$ Merritt, ${ }^{1} \mathrm{~J}$ Wysocki, ${ }^{2} \mathrm{~J}$ Gadzinowski, ${ }^{4} \mathrm{~T}$ Ptaszynski, ${ }^{5} \mathrm{~K}$ Drews. ${ }^{1}$ Department of Preventive Medicine, Poznan University of Medical Sciences, Poznan, Poland; '2Department of Neonatology, Poznan University of Medical Sciences, Poznan, Poland; ${ }^{3}$ Department of Neonatology, Loma Linda University School of Medicine, Loma Linda, USA; ${ }^{4}$ Department of Foundations of Psychological Research, Institute of Psychology Adam Mickiewicz University, Poznan, Poland; ${ }^{5}$ Division of Perinatology and Women's Diseases, Poznan University of Medical Sciences, Poznan, Poland

\subsection{6/archdischild-2014-307384.1291}

Background and aim Nutritional status of the mother in the periconceptional and gestational period can influence the course of pregnancy and newborn health. The aim of our study was to identify and assess the prevalence of disordered eating attitudes in mothers of newborns requiring neonatal intensive care admission compared to those of mothers who delivered healthy infants requiring only normal care in a large maternity hospital.

Methods An anonymous self-report study was conducted among 199 mothers of newborns hospitalised in a Neonatal Intensive Care Unit, and a control group of 127 mothers of healthy newborns who "roomed in" with their mothers. An EAT-26 (Eating Attitudes Test-26) questionnaire and a survey regarding the pregnancy, neonatal outcomes and other health related behaviours were used in the study.

Results Women with EAT-26 scores $>20$ smoked significantly more often during their last pregnancy in the study group ( $\mathrm{p}=$ $0,010)$. There were fewer women with appropriate pre-gestational BMI in the study group $(\mathrm{p}=0.052)$ and they gained less weight during pregnancy $(p=0.001)$. Women who feared weight gain during pregnancy were younger $(p<0.001)$ and had higher EAT-26 scores $(\mathrm{p}<0.001)$. Caesarean section was more frequent in the study group ( $\mathrm{p}=0.017$ ).

Conclusions Perinatal public health education must focus on issues related to eating disorders since the awareness of these issues among obstetricians may improve the outcomes of pregnancy and newborns' health.

\section{PO-0651 ARE NEONATAL AUTOPSY RATES INFLUENCED BY THE TIME OF DEATH OF THE INFANT?}

F Tamanaha, R Fuksman, A Pedraza, L Prudent. Neonatology, Clinica y Maternidad Suizo Argentina, Caba, Argentina

\subsection{6/archdischild-2014-307384.1292}

Background and aims In a previous study on 135 cases, the rate of autopsies in infants dying after 28 days of life appeared low. The aim of the present study was to analyse in a larger sample whether age at death influences autopsy rates.

Methods Retrospective cohort study. Descriptive statistics was used for continuous variables. To study the relationship between rates of autopsy and days of age at death, $\mathrm{Chi}^{2}$ test and Kruskal Wallis rank test were performed. The variable "days of age at death" was stratified in four groups: A: 0-2 days, B: 3-7 days, C: 8-28 days, D: $>28$ days. 